\title{
Railway Track Transition Zones: Design, Construction, Monitoring and Numerical Modelling
}

\author{
E. Fortunato ${ }^{1}$, A. Paixão ${ }^{1}$ and R. Calçada ${ }^{2}$ \\ ${ }^{1}$ Transportation Department \\ National Laboratory for Civil Engineering, Lisbon, Portugal \\ ${ }^{2}$ Department of Civil Engineering \\ Faculty of Engineering, University of Porto, Portugal
}

\begin{abstract}
Railway tracks frequently show higher degradation rates at transition zones from earthworks to bridges, or to other structures. In order to guarantee the safety and comfort of passengers, this aspect results in higher maintenance costs and disturbs railway operations. The behaviour of transition zones is rather complex and its poor performance has been mainly attributed to variations in the vertical stiffness of the track and to differential settlements. These two aspects amplify the dynamic loads of the trains and cause further track degradation.

The Portuguese railway network manager has reported the poor structural behaviour of a few transition zones in some of its lines, which constrain train operations and present high maintenance costs. Thus, a research project is currently underway aiming at contributing to a better understanding of the phenomena that occur at transitions, and at optimizing their design for conventional and high-speed railway lines. In this article, we present some aspects of this research project, namely: a review on the design of backfills for transition zones; a comparison between numerical models found in the literature that were used to study the problem; some results obtained during the construction (characterization of materials and layers) and operation of the line.
\end{abstract}

Keywords: transition zones, backfills, numerical modelling, cyclic load triaxial tests, field characterization, track monitoring, high-speed railway lines.

\section{Introduction}

It is known that the railway track deteriorates under external factors, such as train loads. Moreover, there are some critical locations that evidence higher degradation rates, both in conventional and high-speed railway lines (HSL), consequently requiring more maintenance to guarantee passenger's safety and comfort. These critical locations are usually a consequence of discontinuities and faults found in the 